\title{
Efeito da lavagem e da adição de aditivos sobre a estabilidade de carne mecanicamente separada de tilápia do Nilo (Oreochromis niloticus) durante estocagem a $-18^{\circ} \mathrm{C}$
}

\author{
Effect of washing and increase of additives on minced stability of Nile tilapia
}

(Oreochromis niloticus) during storage under $-18{ }^{\circ} \mathrm{C}$

\author{
Peter Gaberz KIRSCHNIK ${ }^{1 *}$, Elisabete Maria MACEDO-VIEGAS ${ }^{2}$
}

\begin{abstract}
Resumo
Este estudo teve como objetivo avaliar a influência da lavagem e da adição de eritorbato de sódio e tripolifosfato de sódio na estabilidade de Carne Mecanicamente Separada (CMS) de tilápia de Nilo (Oreochromis niloticus) durante 6 meses de armazenamento a $-18^{\circ} \mathrm{C}$. A CMS obtida por meio de máquina separadora de carne e ossos foi dividida em quatro tratamentos (CMS lavada com e sem aditivos, e CMS não lavada com e sem aditivos) e mantida sob congelamento a $-18{ }^{\circ} \mathrm{C}$, por 180 dias. A estabilidade foi avaliada por meio de análises microbiológicas e determinações de nitrogênio não proteico (NNP), bases nitrogenadas voláteis (BNV), substâncias reativas ao ácido tiobarbitúrico (TBARS), pH e drip (perda de água no descongelamento). O processo de lavagem causou redução de aproximadamente 41,44 e $66 \%$ nos teores de proteína bruta, lipídios e cinzas, respectivamente, reduzindo também os valores iniciais de NNP, BNV e TBARS. Durante o armazenamento, foram observados aumentos ( $\mathrm{p} \leq 0,05)$ nos teores de NNP, BNV e pH em praticamente todos os tratamentos, exceto na CMS lavada com aditivos, que não apresentou aumentos significativos nos teores de NNP e pH. O uso de aditivos nas CMS diminuiu o drip ao longo do armazenamento, mas não alterou $(\mathrm{p}>0,05)$ os teores de TBARS. Os parâmetros microbiológicos avaliados não ultrapassaram os limites permitidos pela legislação. As CMS permaneceram estáveis e em boas condições de utilização, independentemente da inclusão de aditivo, sendo viável sua estocagem a $-18^{\circ} \mathrm{C}$ por 180 dias.
\end{abstract}

Palavras-chave: tilápia do Nilo; carne mecanicamente separada; armazenamento.

\begin{abstract}
This study evaluates the influence of washing and adding sodium erythorbate and sodium tripolyphosphate on the stability of minced Nile Tilapia (Oreochromis niloticus) during 6 months of storage under $-18^{\circ} \mathrm{C}$. The minced fish was obtained using a meat bone separator and was divided into four treatments (washed minced fish with or without additives and non-washed minced fish with or without additives) and kept at $-18{ }^{\circ} \mathrm{C}$ for 180 days. The stability of the minced fish was evaluated by microbiological analyses and the following parameters nonproteic nitrogen (NNP), total nitrogen volatile bases (BNV), Thiobarbituric Acid Reactive Substances (TBARS), pH, and Drip (loss of water in thaw process) were evaluated. The washing process caused a reduction of approximately 41,44 , and $66 \%$ in total protein, lipid, and ash values, respectively reducing also the initial values of NNP, BNV, and TBARS. During the storage, an increase ( $p>0,05)$ was observed in the $\mathrm{NNP}, \mathrm{BNV}$, and $\mathrm{pH}$ values for all treatments, except for the non-washed minced with additives which did not show statistic increase in the $\mathrm{NNP}$ and $\mathrm{pH}$ values. The use of additives in the minced fish caused a decrease of the drip during the storage, but it did not affect $(\mathrm{p}>0,05)$ the TBARS values. Microbiological parameters do not exceed the values allowed by the legislation. the minced fish was maintained at stable and good conditions for use regardless of the addition of additives, so it can be stored for up 180 days at $-18{ }^{\circ} \mathrm{C}$.
\end{abstract}

Keywords: Nile tilapia; minced; storage.

\section{Introdução}

A aquicultura continental é uma atividade em franca expansão no Brasil. Em 2004, foram produzidas 180.730 toneladas de pescado, apresentando crescimento de $23,55 \%$ referente ao que foi produzido pela aquicultura no ano 2000. Dentre as espécies mais cultivadas, a tilápia do Nilo vem se destacando, em virtude de suas características zootécnicas e de processamento. Em 2000, a produção de tilápia representava cerca de $18,4 \%$ do total da aquicultura, aumentando para 38\% em 2004, com valores próximos a 69.000 toneladas (IBAMA, 2005).
O crescimento das tilápias em cativeiro ocorre de forma heterogênea, de modo que, no momento da despesca encontramse animais abaixo do peso de comercialização. A produção de Carne Mecanicamente Separada (CMS), utilizando tilápias com peso entre 50 e 200 g pode representar uma alternativa tecnologicamente viável para este problema, podendo reduzir os custos de produção. Além disso, representa uma boa opção para a indústria de processamento, pois a CMS de pescado pode ser empregada na confecção de uma variada linha de produtos, tais

Recebido para publicação em 3/9/2007

Aceito para publicação em 4/2/2009 (002821)

Centro de Ciências Agrárias e Ambientais - CCAA, Pontifícia Universidade Católica do Paraná - PUCPR, BR 376, Km 14, CP 129, CEP 83010-500,

São José dos Pinhais - PR, Brasil, E-mail: petergk76@yahoo.com.br

2 Departamento de Zootecnia, Faculdade de Zootecnia e Engenharia de Alimentos, Universidade de São Paulo - USP, Av. Duque de Caxias Norte, 225, Centro,

CEP 13635-900, Pirassununga - SP, Brasil,E-mail: emviegas@usp.br

${ }^{*}$ A quem a correspondência deve ser enviada 
como: fishburger, salsichas, empanados e enlatados, tirinhas de peixe, nuggets, etc. (MARCHI, 1997; SIDDAIAH et al., 2001).

$\mathrm{O}$ processo de lavagem pode melhorar a qualidade e as características funcionais da CMS de pescado (HASSAN; MATHEW, 1999), removendo sangue, pigmentos, proteínas sarcoplasmáticas, componentes solúveis, lipídios e outras substâncias que podem catalisar a degradação proteica, a oxidação lipídica e causar coloração indevida no produto final (ADU et al., 1983; LEE, 1986; BORDERÍAS; TEJADA, 1987; SUZUKI, 1987; OHSHIMA et al., 1993; TENUTA-FILHO; JESUS, 2003). Entretanto, a lavagem conduz à perda de proteína e outros nutrientes solúveis, gerando efluente líquido abundante (OHSHIMA et al., 1993; TENUTA-FILHO; JESUS, 2003). Simões et al. (1998) observaram diminuições nos teores de proteína, lipídios e cinzas de aproximadamente 53, 62 e $89 \%$, respectivamente, durante o processo de lavagem de CMS de pescada olhuda. Adu et al. (1983) relataram perdas de sólidos de aproximadamente 37\% durante a lavagem de CMS de rockfish (Sebastes. sp.), com diminuição dos teores de P, Mg, K e Na nas CMS lavadas. Segundo Eymard et al. (2005), o processo de lavagem remove os pró-oxidantes e antioxidantes solúveis em água, tornando a CMS mais susceptível à oxidação. Entretanto, segundo os autores, o processo de lavagem também remove, significativamente, os produtos gerados pela oxidação lipídica durante a extração da CMS. Gryschek et al. (2003) observaram que, independente da lavagem da CMS de tilápia, estas permaneceram estáveis e próprias para o consumo durante 180 dias de armazenamento a $-16^{\circ} \mathrm{C}$.

A estocagem sob congelamento não interrompe completamente todas as possíveis alterações na qualidade da CMS de pescado. As reações que induzem as alterações oxidativas e a desnaturação proteica continuam a ocorrer, mesmo em baixas temperaturas (KURADE; BARRANOWSKI, 1987; KUHN; SOARES, 2002). A incorporação de antioxidantes e de crioproterores nas CMS de pescado pode melhorar a estabilidade durante o congelamento (ANESE; GORMLEY, 1996; TENUTA-FILHO; JESUS, 2003). Muitas substâncias, incluindo o ácido ascórbico, eritorbato de sódio, polifosfatos, ácido cítrico e sorbitol, entre outras, têm sido avaliadas para controlar a oxidação lipídica e aumentar a estabilidade proteica em CMS de pescados durante o congelamento (RODRÍGUEZ; BELLO, 1987; CASTRO et al., 1997; SULTANBAWA; LI-CHAN, 1998; ABDEL-AAL, 2001; HERRERA; MACKIE, 2004).

O objetivo deste trabalho foi avaliar o efeito da lavagem e da adição de eritorbato e tripolifosfato de sódio sobre a estabilidade de Carne Mecanicamente Separada de tilápia do Nilo, durante armazenagem sob congelamento por 180 dias.

\section{Material e métodos}

\subsection{Obtenção da CMS, armazenamento e amostragem}

Foram utilizadas aproximadamente 400 tilápias do Nilo (Oreochromis niloticus), com peso médio de $136 \mathrm{~g}$ ( $\pm 23 \mathrm{~g}$ ), provenientes de pisciculturas da região de Pirassununga (São Paulo, Brasil). Os peixes foram retirados dos viveiros, lavados com água corrente e abatidos por choque térmico. As tilápias foram então colocadas em caixas térmicas com gelo e levadas ao Laboratório de Processamento de Produtos Aquáticos da PCAPS/USP, em Pirassununga/SP. No laboratório, os peixes foram lavados com água clorada (5 ppm), descabeçados, eviscerados e lavados novamente. Logo após a limpeza, a CMS de tilápia foi extraída em despolpadora mecânica Hi-Tech 250, obtendo-se aproximadamente $25 \mathrm{~kg}$ de CMS.

Após este processamento inicial, a CMS obtida foi dividida em quatro tratamentos. O Tratamento $\mathrm{A}_{1}$ consistiu em CMS não lavada sem aditivos; o Tratamento $\mathrm{A}_{2}$, CMS não lavada com aditivos; Tratamento $\mathrm{B}_{1}$, CMS lavada sem aditivos; e o Tratamento $\mathrm{B}_{2}$, CMS lavada com aditivos. Para todos os tratamentos, foram amostradas porções de $200 \mathrm{~g}$, embaladas em saco de polietileno, congeladas em ultracongelador a $-40{ }^{\circ} \mathrm{C} \mathrm{e}$ estocadas a $-18{ }^{\circ} \mathrm{C}$ durante 6 meses. A CMS dos tratamentos com aditivos receberam quantidades iguais de eritorbato de sódio $(0,1 \%)$ e tripolifosfato de sódio $(0,5 \%)$.

O procedimento de lavagem foi efetuado com água gelada na proporção de 3 L de água para $1 \mathrm{~kg}$ de CMS, agitação durante 2 minutos e repouso por 3 minutos. Logo após, a mistura foi filtrada através de um tecido de nylon para a retirada do excesso de água. Para uma melhor lavagem, este processo foi repetido por duas vezes.

Três amostragens ( \pm 200 g cada $)$ de cada tratamento foram realizadas no início do armazenamento (tempo 0) e a intervalos de 30, 60, 90, 120, 150 e 180 dias de estocagem, para a realização de análises físico-químicas e microbiológicas.

\subsection{Análises físico-químicas}

A leitura do $\mathrm{pH}$ foi feita em peagâmetro VDSF (modelo F-1002) com eletrodo VDSF (modelo 9411), após a homogeneização de $10 \mathrm{~g}$ de CMS com $40 \mathrm{~mL}$ de água destilada. Análises de Nitrogênio de Bases Nitrogenadas Voláteis (N-BNV) foram realizadas de acordo com Howgate (1976); as de Nitrogênio Não-Proteico (NNP), segundo a AOAC (1995); e as de Substâncias Reativas ao Ácido Tiobarbitúrico (TBARS), conforme Vyncke (1970), utilizando-se o Tetrametoxipropano para a obtenção da equação da reta utilizada no cálculo dos valores de TBARS $(y=66,023 x+0,0077)$. Teores de umidade, cinza, extrato etéreo e proteína bruta foram determinados de acordo com AOAC (1995).

O teste de drip, perda de água no descongelamento, foi baseado em Santo et al. (1980). Três amostras congeladas de cada tratamento, com peso de $50 \mathrm{~g}$ cada, foram colocadas individualmente em funis sob tubos de ensaios graduados. Os funis e os tubos foram cobertos com filme plástico para prevenir a evaporação de líquidos, e o conjunto permaneceu durante 48 horas a uma temperatura de $10{ }^{\circ} \mathrm{C}$, sendo o drip medido e calculado em porcentagem de volume em relação ao peso da amostra.

\subsection{Análises microbiológicas}

O desenvolvimento microbiológico foi avaliado por meio das análises de contagem total de psicrotróficos em placas, pela técnica do pour plate; detecção da Salmonella foi realizada seguindo metodologia sugerida pelo Ministério da Agricultura (BRASIL, 1981); detecção de Staphylococcus aureus, de acordo com APHA (1992). Determinações do Número Mais Provável de coliformes termotolerantes e totais foram realizadas segundo APHA (1992). 


\subsection{Análises estatísticas}

O estudo foi realizado em um delineamento inteiramente casualizado com quatro tratamentos e três repetições. As médias foram comparadas pelo teste de Tukey em nível de 5\% de probabilidade. As análises foram realizadas pelo programa SAS (1999).

\section{Resultados e discussão}

O rendimento da extração da CMS de tilápia do Nilo foi de $46,90 \%$ em relação ao peixe inteiro e de $78,60 \%$ em relação ao peixe eviscerado e descabeçado. Os valores reportados neste trabalho são superiores aos encontrados por Gryschek et al. (2003), que observaram rendimentos de 42,56 e 33,57\% em relação ao peixe inteiro e 65,96 e 51,73\%, em relação ao peixe eviscerado e descabeçado, respectivamente para tilápia vermelha (Oreochromis spp.) e tilápias do Nilo (Oreochromis niloticus). Ohshima et al. (1993) relataram rendimento médio de $47 \%$ da extração da CMS de alaska pollock (Theragra chalcogramma), em relação aos peixes inteiros. $\mathrm{O}$ rendimento após o processo de lavagem pode ser influenciado pela eficiência da retirada de água, número de lavagens e tipo de equipamento utilizado. Neste estudo, o rendimento médio após o processo de lavagem foi de $84,7 \%$.

Os valores referentes à composição centesimal da CMS de tilápia estão expostos na Tabela 1. O processo de lavagem propiciou remoção de proteínas hidrossolúveis, minerais e lipídios, tornando a CMS lavada uma matéria-prima diferente da CMS não lavada. Foi observado aumento nos teores de umidade na CMS após o processo de lavagem. Este comportamento também foi observado por outros autores (RODRIGUEZ; BELLO, 1987; GRYSCHEK et al., 2003; NEIVA, 2003). Isto ocorreu, provavelmente, devido ao aumento na capacidade de hidratação das proteínas miofibrilares que estão presentes em grande quantidade na CMS, como consequência da remoção das proteínas sarcoplasmáticas durante a lavagem (SUZUKI, 1987).

Os valores de umidade encontrados nas CMS lavadas neste estudo estão próximos aos observados por Lanfer-Marquez e Mira (2005), entre 79,58 e 88,70\% em amostras de surimi de peixes marinhos de baixo valor comercial (Paralonchurus brasiliensis e Cynoscion virescens). Porém encontram-se abaixo do verificado por Simões et al. (1998), que relataram teores de 92,78\% de umidade em CMS lavada de pescada (Cynoscion striatus). Valores de umidade em CMS lavadas podem diferir muito entre si, dependendo do processo de lavagem e de retirada de água utilizados.

Observou-se diminuição nos teores de proteína nas CMS lavadas, que provavelmente ocorreu devido à remoção da maioria das proteínas solúveis sarcoplasmáticas. Tal fato também foi observado por outros autores (ADU et al., 1983; GRYSCHEK et al., 2003), que reportaram consideráveis perdas de proteína e outros componentes hidrossolúveis após o processo de lavagem de CMS de tilápia e diversas espécies de rockfish (Sebastes sp.).

No presente estudo, o processo de lavagem reduziu significativamente os teores de lipídios, de 2,91\% para 1,63\%, fato também observado por Eymard et al. (2005), cujos valores diminuíram de 3,6\% para 1,2\% após a lavagem de CMS de horse mackerel (Trachurus trachurus). Gryschek et al. (2003) observaram teores de lipídios de 4,23 e 1,70\% em CMS de tilápia do Nilo, não lavada e lavada, respectivamente.

Os teores de cinzas encontrados na CMS não lavada deste trabalho estão próximos ao $1,5 \%$ de cinzas, encontrados por Marchi (1997), em CMS de tilápias do Nilo. Após a lavagem, observou-se uma redução significativa nos teores de cinzas $(65,9 \%)$, indicando grande perda de minerais durante este processo devido à lixiviação ocorrida através da água da lavagem. Marchi (1997) e Gryschek et al. (2003) reportaram reduções de 70 e $80 \%$, respectivamente, nos teores de cinzas após o processo de lavagem em CMS de tilápia do Nilo. Segundo Lee (1986), a grande perda de sólidos (25 a 40\%) que ocorre durante as operações de lavagens se deve à perda de lipídios e cinzas.

Observou-se diminuição $(\mathrm{p} \leq 0,05)$ nos teores de NNP após o processo de lavagem da CMS (Tabela 2), fato que pode ser atribuído à lixiviação dos compostos nitrogenados solúveis formadores do NNP, que ocorre durante o processo (HASSAN; MATHEW, 1999). Valores de NNP ao redor de 344 mg NNP.100 g-1 de músculo de tilápia do Nilo foram relatados por Contreras-Guzmán (2002). Os teores de NNP nas CMS não lavadas, com e sem aditivos, foram semelhantes entre si, apresentando aumento significativo ( $\mathrm{p} \leq 0,05)$ ao longo da estocagem de 180 dias. Isto pode ser atribuído à hidrólise de proteínas por enzimas bacterianas ou por proteases musculares (CONTRERAS-GUZMÁN, 2002).

Abdel-aal (2001), estudando CMS de Karmout (Claries lazera) estocada a $-18^{\circ} \mathrm{C}$ por 180 dias, relatou aumento no teor de NNP ao longo da estocagem, com valores iniciais de 276,4 mg. $100 \mathrm{~g}^{-1}$ e finais de 403,2 mg. $100 \mathrm{~g}^{-1}$ de CMS. Neste estudo, CMS lavadas com e sem aditivos apresentaram teores de NNP bem menores que a CMS não lavada, durante o armazenamento, indicando que este processo retirou grande parte das enzimas e proteases musculares que poderiam hidrolisar as proteínas e aumentar os valores de NNP.

A produção de BNV durante a estocagem do pescado é resultante da ação de enzimas dos tecidos e da atividade microbiológica, sendo composta principalmente por amônia, trimetilamina, dimetilamina e, provavelmente, por traços de monometilamina e propilamina, que se formariam em etapas mais avançadas de decomposição (CONTRERAS-GUZMÁN, 2002). Os teores de $\mathrm{BNV}$ encontrados nos diferentes tratamentos para CMS ao longo

Tabela 1. Composição centesimal das CMS lavadas e não lavadas de tilápia do Nilo.

\begin{tabular}{ccccc}
\hline CMS & Umidade (\%) & Proteína bruta (\%) & Lipídios (\%) & Cinzas (\%) \\
\hline CMS não lavada & $79,83 \pm 0,35^{\mathrm{B}}$ & $15,13 \pm 0,17^{\mathrm{A}}$ & $2,91 \pm 0,32^{\mathrm{A}}$ & $1,35 \pm 0,04^{\mathrm{A}}$ \\
CMS lavada & $88,78 \pm 0,19^{\mathrm{A}}$ & $8,93 \pm 0,22^{\mathrm{B}}$ & $1,63 \pm 0,07^{\mathrm{B}}$ & $0,46 \pm 0,02^{\mathrm{B}}$ \\
\hline
\end{tabular}

Médias $(n=3)$ de análises em triplicata; médias dos mesmos parâmetros nas mesmas colunas, seguidas por letras maiúsculas distintas, diferem significativamente entre si pelo teste de Tukey $(\mathrm{p} \leq 0,05)$ 
de 180 dias de estocagem estão mostrados na Tabela 3. Os menores teores iniciais de BNV observados nas CMS lavadas, quando comparadas com as CMS não lavadas podem ser justificados pela provável perda de compostos nitrogenados voláteis durante $\mathrm{o}$ processo de lavagem. Diminuição nos teores de BNV durante o processo de lavagem também foram observadas por Rodríguez e Bello (1987) e Neiva (2003), que constataram diminuição de 83,3 e $34,8 \%$ de BNV após o processo de lavagem de CMS de sardinha (Sardinella brasiliensis) e CMS de peixes da fauna acompanhante de pesca de camarão, respectivamente.

$\mathrm{O}$ teor de BNV nas CMS não lavadas, com e sem aditivos, apresentaram comportamento semelhantes, tendo aumentos significativos ( $\mathrm{p} \leq 0,05)$ após 30 dias de estocagem, com pequenas oscilações até o final do período. Nas CMS lavadas com e sem aditivos, os teores de BNV aumentaram $(\mathrm{p} \leq 0,05)$ somente após 120 dias de estocagem, atingindo valores um pouco maiores para a CMS sem aditivos $\left(3,02 \mathrm{mg} .100 \mathrm{~g}^{-1}\right)$ do que para a CMS com aditivos (2,63 mg.100 $\left.\mathrm{g}^{-1}\right)$ aos 180 dias. Os pequenos aumentos observados nos teores de BNV nas CMS estudadas indicam que os processos de congelamento e de armazenamento aos quais as CMS foram submetidas inibiram ou limitaram o desenvolvimento da atividade microbiológica e a ação de enzimas endógenas.

Aumentos mais expressivos de BNV foram observados por Siddaiah et al. (2001) em CMS de carpas prateadas (Hypophthalmichthys molitrix) armazenadas a $-18^{\circ} \mathrm{C}$, relatando aumento de 1,98 para $32,85 \mathrm{mg} .100 \mathrm{~g}^{-1}$ ao final do período de 180 dias de estocagem. Resultados similares foram relatados por Reddy et al. (1992), com aumentos nas BNV de 4,0 para $18,4 \mathrm{mg} .100 \mathrm{~g}^{-1}$ ao final do período de 180 dias de estocagem congelada de CMS de perca rosa (Nemipterus japonicus). Entretanto, Jesus et al. (2001), avaliando a estabilidade química e microbiológica de CMS congeladas de peixes amazônicos, observaram tendência decrescente $(\mathrm{p}>0,05)$ das BNV ao longo da estocagem de 150 dias, justificando este comportamento devido à diminuição da atividade microbiana durante este período. O valor máximo de BNV observado nas CMS estudadas permaneceu abaixo de $30 \mathrm{mg} \mathrm{BNV/100} \mathrm{g,} \mathrm{que} \mathrm{é} \mathrm{o} \mathrm{limite} \mathrm{estabelecido} \mathrm{pela} \mathrm{legislação} \mathrm{de}$ pescados e derivados para efeito de consumo (BRASIL, 1997).

Os resultados dos valores de $\mathrm{pH}$ nas CMS podem ser observados na Figura 1. Em geral, os valores de $\mathrm{pH}$ aumentaram significativamente $(\mathrm{p} \leq 0,05)$ durante os primeiros 60 dias de estocagem, permanecendo, então, praticamente constantes até o fim do período. $\mathrm{O}$ aumento do $\mathrm{pH}$ pode indicar degradação proteica, com produção de substâncias como amônia e outras aminas. Rodríguez e Bello (1987), trabalhando com CMS congelada de peixes da fauna acompanhante da pesca de camarões, estocada a -10 e $-30^{\circ} \mathrm{C}$, durante 180 dias, verificaram que não ocorreram alterações significativas nos valores de $\mathrm{pH}$ em ambas as temperaturas. Entretanto, Jesus et al. (2001) encontraram

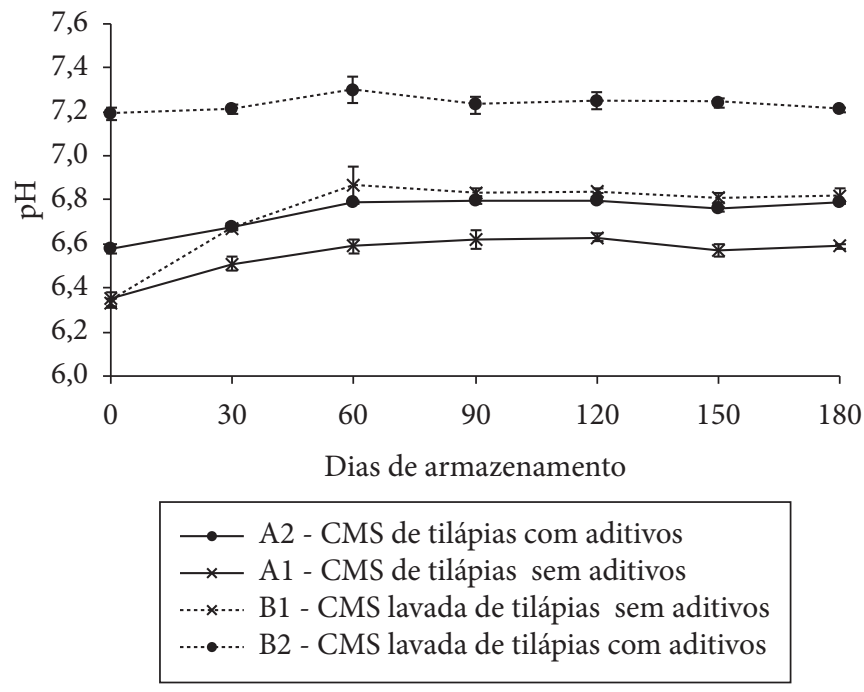

Figura 1. Valores de $\mathrm{pH}$ das CMS de tilápia do Nilo lavadas e não lavadas, com e sem aditivos, durante armazenamento.

Tabela 2. Nitrogênio não proteico ( $\mathrm{mg} \mathrm{N} 100 \mathrm{~g}^{-1}$ ) nas CMS de tilápia do Nilo lavadas e não lavadas, com e sem aditivos durante armazenamento.

\begin{tabular}{|c|c|c|c|c|c|c|c|}
\hline \multirow[t]{2}{*}{ Tratamentos CMS } & \multicolumn{7}{|c|}{ Dias de armazenamento } \\
\hline & 0 & 30 & 60 & 90 & 120 & 150 & 180 \\
\hline $\mathrm{A}_{2}$ & $299,7^{\mathrm{Ba}} \pm 2,8$ & $296,5^{\mathrm{Ba}} \pm 5,0$ & $300,0^{\mathrm{Ba}} \pm 4,4$ & $300,5^{\mathrm{Ba}} \pm 11,7$ & $296,6^{\mathrm{Ba}} \pm 3,4$ & $295,0^{\mathrm{Ba}} \pm 4,1$ & $319,7^{\mathrm{Aa}} \pm 1,7$ \\
\hline $\mathrm{B}_{2}$ & $50,5^{\mathrm{Ab}} \pm 2,4$ & $50,5^{\mathrm{Ab}} \pm 2,4$ & $47,3^{\mathrm{Ab}} \pm 2,4$ & $48,9^{\mathrm{Ab}} \pm 3,7$ & $45,7^{\mathrm{Ab}} \pm 1,7$ & $51,3^{\mathrm{Ab}} \pm 3,4$ & $51,5^{\mathrm{Ab}} \pm 4,2$ \\
\hline
\end{tabular}

Médias ( $\mathrm{n}=3$ ) de análises; $\mathrm{A}_{1}=\mathrm{CMS}$ não lavada sem aditivo, $\mathrm{A}_{2}=\mathrm{CMS}$ não lavada com aditivos, $\mathrm{B}_{1}=\mathrm{CMS}$ lavada sem aditivo, $\mathrm{B}_{2}=\mathrm{CMS}$ lavada com aditivos; médias nas mesmas linhas seguidas por letras Maiúsculas distintas diferem significativamente pelo teste de Tukey ( $\mathrm{p} \leq 0,05)$; e médias nas mesmas colunas, seguidas de letras minúsculas distintas, diferem significativamente pelo teste de Tukey $(\mathrm{p} \leq 0,05)$

Tabela 3. Bases nitrogenadas voláteis ( $\left.\mathrm{mg} \mathrm{N} 100 \mathrm{~g}^{-1}\right)$ nas CMS de tilápia do Nilo lavadas e não lavadas, com e sem aditivos durante armazenamento.

\begin{tabular}{cccccccc}
\hline Tratamentos CMS & \multicolumn{7}{c}{ Dias de armazenamento } \\
\cline { 2 - 7 } & 0 & 30 & 60 & 90 & 120 & 150 \\
\hline $\mathrm{A}_{1}$ & $8,63^{\mathrm{Ca}} \pm 0,44$ & $8,88^{\mathrm{BCa}} \pm 0,32$ & $10,47^{\mathrm{Aa}} \pm 0,33$ & $9,66^{\mathrm{ABCa}} \pm 1,56$ & $10,60^{\mathrm{Aa}} \pm 0,4$ & $9,93^{\mathrm{ABa}} \pm 0,07$ & $10,03^{\mathrm{ABa}} \pm 0,29$ \\
$\mathrm{~A}_{2}$ & $8,37^{\mathrm{Ca}} \pm 0,76$ & $8,56^{\mathrm{Ca}} \pm 0,1$ & $10,85^{\mathrm{Ba}} \pm 0,33$ & $8,23^{\mathrm{Ca}} \pm 0,41$ & $11,99^{\mathrm{Ab}} \pm 0,0$ & $9,99^{\mathrm{Ba}} \pm 0,10$ & $10,28^{\mathrm{Ba}} \pm 0,33$ \\
$\mathrm{~B}_{1}$ & $0,26^{\mathrm{Cb}} \pm 0,45$ & $0,26^{\mathrm{Cb}} \pm 0,40$ & $0,42^{\mathrm{Cb}} \pm 0,37$ & $0,61^{\mathrm{Cb}} \pm 0,06$ & $1,32^{\mathrm{Bb}} \pm 0,15$ & $2,44^{\mathrm{Ab}} \pm 0,28$ & $3,02^{\mathrm{Ab}} \pm 0,34$ \\
$\mathrm{~B}_{2}$ & $0,26^{\mathrm{Cb}} \pm 0,44$ & $0,26^{\mathrm{Cb}} \pm 0,11$ & $0,77^{\mathrm{BCb}} \pm 0,19$ & $0,66^{\mathrm{BCb}} \pm 0,09$ & $1,12^{\mathrm{Bb}} \pm 0,20$ & $2,12^{\mathrm{Ab}} \pm 0,90$ & $2,63^{\mathrm{Ab}} \pm 0,22$ \\
\hline
\end{tabular}

Médias $(\mathrm{n}=3)$ de análises em triplicata; $\mathrm{A}_{1}=\mathrm{CMS}$ não lavada sem aditivo; $\mathrm{A}_{2}=\mathrm{CMS}$ não lavada com aditivos; $\mathrm{B}_{1}=\mathrm{CMS}$ lavada sem aditivo; $\mathrm{B}_{2}=\mathrm{CMS}$ lavada com aditivos; e médias nas mesmas linhas seguidas por letras Maiúsculas distintas diferem significativamente pelo teste de Tukey ( $\mathrm{p} \leq 0,05)$ entre si. Médias nas mesmas colunas, seguidas de letras minúsculas distintas, diferem significativamente pelo teste de Tukey $(\mathrm{p} \leq 0,05)$ entre si. 
aumentos nos valores de $\mathrm{pH}$ de 6,50 para até 7,07 em CMS de peixes amazônicos estocada por 150 dias sob congelamento. $\mathrm{O}$ comportamento do $\mathrm{pH}$ durante o armazenamento, sob congelamento, é dependente da temperatura de estocagem, composição em sais, estado fisiológico, poder tampão das proteínas e ação enzimática (GRYSCHEK et al., 2003). Neste estudo, o processo de lavagem provavelmente alterou a composição de sais e enzimas presentes na CMS e provocou mudanças no $\mathrm{pH}$ durante o período de armazenamento.

A adição de tripolifosfato de sódio e de eritorbato de sódio nas CMS do presente estudo elevou seus teores de $\mathrm{pH}$. A adição de polifosfatos ao surimi de pescado melhorou sua qualidade durante estocagem sob congelamento, devido ao seu efeito crioprotetor e à propriedade de manter $\mathrm{oH}$ próximo à neutralidade (KONNO, 1992). Tendo em vista os valores próximos de $\mathrm{pH}$ observados nas CMS lavadas sem aditivos e não lavadas com aditivos, pode-se sugerir que o processo de lavagem teria o mesmo efeito que a adição de tripolifosfato na CMS sem lavar. Talvez, como uma forma de diminuir efluentes gerados pela lavagem, o tripolifosfato possa ser utilizado na CMS como forma alternativa de manter o $\mathrm{pH}$ em valores aceitáveis, de acordo com a legislação (BRASIL, 1997).

Os valores do TBARS são utilizados como indicador do grau de oxidação lipídica, quantificando o malonaldeído, que é um dos principais produtos formados durante o processo oxidativo. Todas as CMS estudadas mostraram um gradual aumento nos valores de TBARS ao longo da estocagem. Foram observados teores significativamente menores de TBARS nas CMS lavadas quando comparadas com as não lavadas (Figura 2). Este fato pode ser explicado devido à remoção, durante o processo de lavagem, da maior parte dos produtos primários e secundários da oxidação lipídica (EYMARD et al., 2005). O processo de lavagem também promove a remoção de pigmentos, sangue, lipídios e compostos heme, que causam a oxidação lipídica (TENUTA FILHO; JESUS, 2003). Isto justifica os menores

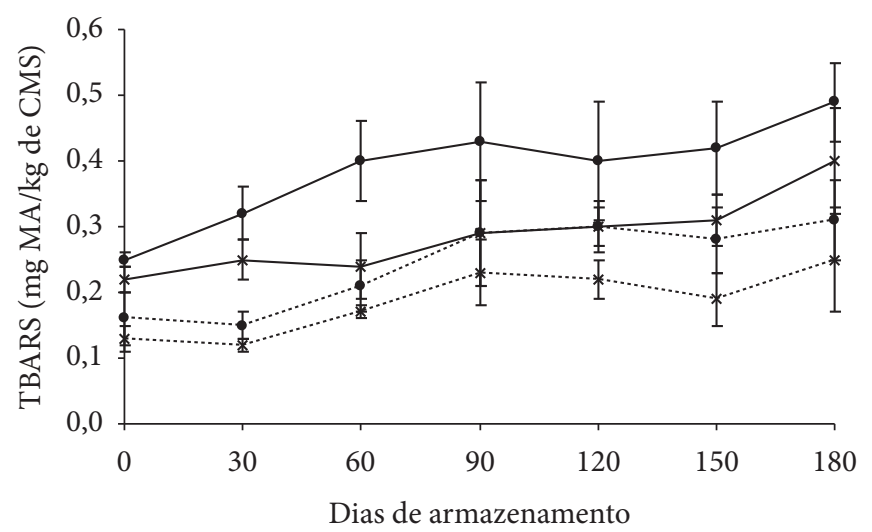

$$
\begin{aligned}
& \longrightarrow \multimap \text { A2 - CMS de tilápias com aditivos } \\
& \rightarrow \text { A1 - CMS de tilápias sem aditivos } \\
& \cdots \cdots \cdots \text { B1 - CMS lavada de tilápias sem aditivos } \\
& \cdots \cdots \cdots \text { B2 - CMS lavada de tilápias com aditivos }
\end{aligned}
$$

Figura 2. Valores de TBARS (mg de aldeído malônico/kg) nas CMS de tilápia do Nilo lavadas e não lavadas, com e sem aditivos, durante armazenamento. valores de TBARS observados nas CMS lavadas (0,25 mg de malonaldeído $/ \mathrm{kg}$ ) ao final do período de estocagem.

Neste estudo, o maior valor de TBARS observado após 180 dias de estocagem foi $0,49 \mathrm{mg}$ de malonaldeído/kg na CMS não lavada com aditivos. Segundo Al-Kahtani et al. (1996), o pescado pode ser considerado em bom estado de consumo, quando apresentar valores abaixo de $3 \mathrm{mg}$ de malonaldeído $/ \mathrm{kg}$ de amostra. Os baixos valores de TBARS observados podem ser atribuídos ao pequeno teor de lipídio encontrado nas CMS (Tabela 1), indicando que a rancidez oxidativa não se constituiu em um fator determinante para a redução de qualidade do produto. Jesus et al. (2001), estudando a estabilidade de CMS de mapará (Hypophthalmus edentatus) sob congelamento, também observaram pequenas variações nos valores de TBARS durante a estocagem, com valores iniciais de 0,22 e finais de 0,64 mg de malonaldeído $/ \mathrm{kg}$.

A adição de eritorbato de sódio nas CMS lavadas e não lavadas não teve efeito significativo $(\mathrm{p} \leq 0,05)$ sobre os valores do TBARS durante a estocagem. Neiva (2003) não constatou alterações nos valores de TBARS durante a estocagem sob congelamento de CMS lavadas de sardinha com ou sem a adição de TBHQ, $\alpha$-tocoferol e eritorbato de sódio, concluindo que a embalagem a vácuo foi suficiente para manter a estabilidade lipídica.

Durante o descongelamento e a cocção, ocorrem perdas de líquido (drip) que contêm vitaminas e minerais, o que resulta em uma perda no valor nutritivo e de qualidade sensorial, tornando os produtos secos e rígidos (GONÇALVES, 2004). Os valores do drip durante o descongelamento ao longo da estocagem podem ser observados na Figura 3. A CMS não lavada sem aditivos apresentou aumento significativo $(\mathrm{p} \leq 0,05)$ nos valores do drip após 60 dias de estocagem. Os valores praticamente constantes do drip na CMS não lavada com aditivos indicam que a incorporação de $0,5 \%$ de tripolifosfato de sódio impediu o aumento


Figura 3. Drip (\%) das CMS de tilápia do Nilo lavadas e não lavadas, com e sem aditivos, durante armazenamento. 
da perda de água no descongelamento. A CMS lavada sem aditivos apresentou maiores valores de $\operatorname{drip}(\mathrm{p} \leq 0,05)$ que as CMS não lavadas sem aditivo, possivelmente devido ao maior teor de umidade inicial observado (Tabela 1). Nas CMS lavadas com e sem aditivos, a perda de drip permanece praticamente constante $(\mathrm{p}>0,05)$ ao longo do período. Entretanto, a adição de $0,5 \%$ de tripolifosfato de sódio nas CMS diminuiu significativamente a formação do drip durante o descongelamento. Segundo Gonçalves (2004), os fosfatos têm a propriedade de devolver às proteínas sua capacidade de retenção de água, mantendo assim a umidade natural do produto, levando a uma perda mínima de água durante o armazenamento por congelamento, no descongelamento e na cocção.

A diminuição da capacidade de retenção de água na CMS está diretamente ligada com o grau de desnaturação das proteínas durante o armazenamento, e os tripolifosfatos melhoram significativamente esta capacidade. Os tripolifosfatos têm a propriedade de afetar a estrutura das proteínas por três vias: aumentando o $\mathrm{pH}$, a força iônica e através de sua interação com as proteínas (RODRÍGUEZ; BELLO, 1987).

Reddy et al. (1992), estudando a estabilidade na estocagem a $-18^{\circ} \mathrm{C}$ de CMS de perca rosa (Nemipterus japonicus) durante 180 dias, observaram aumento do drip ao longo do período, sendo encontrados valores de $4,8 \%$ aos 30 dias e $20 \%$ ao fim do período. Os autores relataram que todas as propriedades funcionais das CMS foram afetadas pela perda de solubilidade das proteínas, que é depende da desnaturação e agregação das estruturas proteicas originais. Abdel-aal (2001) observou que a adição de $0,5 \%$ de tripolifosfato de sódio manteve alta a capacidade de retenção de água durante a estocagem de 180 dias a $-18{ }^{\circ} \mathrm{C}$ de CMS de Karmout (Claries lazera). O autor relatou que este aumento foi devido ao aumento do pH das CMS após a adição do tripolifosfato de sódio.

A contagem total de psicrotróficos permaneceu com valores semelhantes ao longo da estocagem nas CMS não lavadas com e sem aditivos (Tabela 4). Nas CMS lavadas com e sem aditivos, os valores aumentaram $(\mathrm{p} \leq 0,05)$ durante os primeiros 90 dias e, após esse período, diminuíram até o final da estocagem. Contagens de psicrotróficos iniciais mais elevadas foram reportadas por Marchi (1997) e Hassan e Mathew (1999), os quais encontraram contagem total de psicrotróficos de $\log 5,53$ e 3,3 UFC. ${ }^{-1}$, respectivamente, em CMS lavadas de tilápias do Nilo. Rodríguez e Bello (1987), avaliando a

Tabela 4. Contagem total em placas de psicrotróficos (log UFC.g $\mathrm{g}^{-1}$ ) avaliados nas CMS de tilápia do Nilo lavadas e não lavadas, com e sem aditivos durante armazenamento.

\begin{tabular}{cccc}
\hline Tratamentos CMS & \multicolumn{3}{c}{ Dias de armazenamento } \\
\cline { 2 - 4 } & 0 & 90 & 180 \\
\hline $\mathrm{A}_{1}$ & $2,54 \pm 0,4^{\mathrm{A}}$ & $3,37 \pm 0,2^{\mathrm{A}}$ & $2,77 \pm 0,3^{\mathrm{A}}$ \\
$\mathrm{A}_{2}$ & $2,62 \pm 0,5^{\mathrm{A}}$ & $3,11 \pm 0,1^{\mathrm{A}}$ & $3,01 \pm 0,1^{\mathrm{A}}$ \\
$\mathrm{B}_{1}$ & $1,42 \pm 0,4^{\mathrm{B}}$ & $2,20 \pm 0,2^{\mathrm{A}}$ & $1,49 \pm 0,2^{\mathrm{B}}$ \\
$\mathrm{B}_{2}$ & $1,44 \pm 0,2^{\mathrm{B}}$ & $1,56 \pm 0,1^{\mathrm{A}}$ & $1,10 \pm 0,2^{\mathrm{B}}$ \\
\hline
\end{tabular}

Médias ( $\mathrm{n}=3$ ) de análises em triplicata; $\mathrm{A}_{1}=\mathrm{CMS}$ não lavada sem aditivo; $\mathrm{A}_{2}=\mathrm{CMS}$ não lavada com aditivos; $\mathrm{B}_{1}=\mathrm{CMS}$ lavada sem aditivo; $\mathrm{B}_{2}=\mathrm{CMS}$ lavada com aditivos; e médias nas mesmas linhas, seguidas por letras maiúsculas distintas, diferem significativamente entre si pelo teste de Tukey $(\mathrm{p} \leq 0,05)$. estocagem a $-10 \mathrm{e}-30{ }^{\circ} \mathrm{C}$ de CMS de peixes da fauna acompanhante da pesca de camarão, observaram diminuição nas contagens de psicrotróficos ao longo da estocagem de 180 dias, em ambas as temperaturas.

Foi observada menor contagem total de psicrotróficos nas CMS lavadas, sugerindo que o processo de lavagem pode exercer um efeito benéfico de remoção de microrganismos. Tal efeito também foi constatado por Gryschek (2001).

Os resultados da contagem total de psicrotróficos observados nas CMS mantiveram-se abaixo do limite permitido ( $\log 7,0$ UFC. ${ }^{-1}$ ) pela ICMSF (1986), para contagem padrão em placas de microrganismos aeróbicos. Não foi constatada a presença de coliformes fecais, Salmonella e Staphylococcus aureus nas CMS durante o período de estocagem, estando elas dentro dos padrões estabelecidos pela legislação brasileira (BRASIL, 2001).

\section{Conclusões}

A Carne Mecanicamente Separada elaborada com tilápia do Nilo abaixo do peso de abate é uma alternativa viável para o aproveitamento de peixes sem o peso ideal. A adição de tripolifosfato de sódio à carne mecanicamente separada da tilápia do Nilo melhorou a capacidade de retenção de água. Independentemente do processo de lavagem ou da adição de aditivos, as CMS mantiveram-se estáveis durante o período de estocagem a $-18^{\circ} \mathrm{C}$ por 180 dias. Os parâmetros químicos, físicos e microbiológicos avaliados permaneceram constantes ou apresentaram, em alguns casos, apenas pequenos aumentos ao longo da estocagem, não afetando a qualidade das CMS.

\section{Agradecimentos}

Os autores agradecem ao Conselho Nacional de Desenvolvimento Científico e Tecnológico - CNPq, pela concessão da bolsa de doutorado do primeiro autor e pelo apoio financeiro por meio do edital CNPq/CTAGRO/SEAP, processo no 50.4027/03-4.

\section{Referências bibliográficas}

ABDEL-AAL, H. Using antioxidants for extending the shelf life of frozen Nile karmout (Claries lazera) fish mince. Journal of Aquatic Food Product Technology, v. 10, n. 4, p. 87-99, 2001.

ADU, G. A.; BABBITT, J. K.; CRAWFORD, D. L. Effect of washing on the nutritional and quality characteristics of dried minced rochfish flesh. Journal of Food Science, v. 48, p. 1053-1055, 1983.

AL-KAHTANI, H. A.; ABU-TARBOUSH, H. M.; BAJABER, A. S. Chemical changes after irradiation and post-irradiation storage in Tilapia and Spanish mackerel. Journal of Food Science, v. 61, n. 4, p. 729-733, 1996.

ANESE, M.; GORMLEY, R. Effects of dairy ingredients on some chemical, physico-chemical and functional properties of minced fish during freezing and frozen storage. Lebensmittel-Wissenschaft und. Techologie, v. 29, p. 151-157, 1996.

APHA. American Public Health Association. Compendium of methods for the microbiological examination of foods. 3 ed., Washington, 1992.

ASSOCIATION OF OFFICIAL ANALYTICAL CHEMISTS. Official methods of analysis. 16. ed. Arlington: AOAC, 1995. $2 \mathrm{v}$. 
BORDERIAS, A. J.; TEJADA, D. El “surimi”. Revista de Agroquímica y Tecnología de Alimentos, v. 27, n. 1, p. 1-14, 1987.

BRASIL. Ministério da Agricultura e do Abastecimento. Secretaria Nacional de Defesa Agropecuária. Laboratório Nacional de Referência Animal - LANARA. Métodos analíticos para o controle de produtos de origem animal e seus ingredientes: métodos microbiológicos. Brasília, 1981.

BRASIL. Ministério da Agricultura, Pecuária e Abastecimento. Legislação de Pescado e Derivados, Brasília, 1997. Portaria n.185, de 13 de maio de 1997 - Diário Oficial da União 19 de maio de 1997.

BRASIL. Ministério da Saúde. ANVISA, Agência Nacional de Vigilância Sanitária, Brasília, 2001. Resolução RDC n. 12, de 02 de janeiro de 2001 - Diário Oficial da União 10 de janeiro de 2001, seção 1.

CASTRO, M. A. M.; GBMEZ-GUillih, M. C.; MONTERO, P. Influence of frozen storage on textural propertiesof sardine (Sardina pilchardus) mince gels. Food Chemistry, v. 60, n. 1, p. 85-93, 1997.

CONTRERAS-GUZMÁN, E. S. Bioquímica de pescados e invertebrados. Santiago: CECTA-USACH, 2002, 309 p.

EYMARD, S.; CARCOUET, E.; ROCHET, M. J.; DUMAY, J.; CHOPIN, C.; GENOT, C. Development of lipid oxidation during manufacturing of horse mackerel surimi. Journal of the Science of Food and Agriculture, v. 85, p. 1750-1756, 2005.

GONÇALVES, A. A. Los fosfatos en el pescado: ¿fraude o mejora de la calidad? Revista INFOPESCA, n.20, out/dez, p.19-28, 2004.

GRYSCHEK, S. F. B. Obtenção, caracterização e estabilidade ao congelamento de minces elaborados com tilápia nilótica (Oreochromis niloticus) e tilápia vermelha (Oreochromis spp). Piracicaba, 2001, 84p. Dissertação (Mestrado em Ciência e Tecnologia de Alimentos) - Escola Superior de Agricultura Luiz de Queiroz (ESALQ).

GRYSCHEK, S. F. B.; OETTERER, M.; GALLO, C. R. Characterization and frozen storange stability of minced Nile Tilapia (Oreochromis niloticus) and red tilapia (Oreochromis spp.). Journal os Aquatic Food Product Techology, v. 12, n. 3, p. 57-69, 2003.

HASSAN, F. e MATHEW, S. Physico-chemical, microbiological and sensory characteristics of washed fish mince prepared from some selected species of fish. Journal of Food Science and Technology, v. 36, n. 5, p. 459-462, 1999.

HERRERA, J. R., MACKIE, I. M. Cryoprotection of frozen-stored actomyosin of farmed rainbow trout (Oncorhynchus mykiss) by some sugars and polyols. Food Chemistry. v. 84, p. 91-97, 2004.

HOWGATE, P. Determination of total volatile bases. Aberdeen: Torry Research Station.1976, TD 564, Appendix 4.

IBAMA. Instituto Brasileiro do Meio Ambiente e dos Recursos Naturais Renováveis. Estatística da Pesca 2004, grandes regiões e unidades da confederação. 2005, p.136.

ICMSF - International Commission on Microbiological Specification for Foods. Microorganisms in foods. 2-Sampling for microbiological analysis: principles and specific applications. Toronto: University of Toronto Press, 1986. 193 p.

JESUS, R. S.; LESSI, E.; TENUTA-FILHO, A. Estabilidade química e microbiológica de minced fish de peixes amazônicos durante o congelamento. Ciência e Tecnologia de Alimentos, v. 21, n. 2, p. 144-148, 2001.

KONNO, K. Suppression of thermal denaturation of miosin subfragment-1 of Alaska Pollack by sorbitol and accelerated inactivation by pyrophosphate. Journal Food Science, v. 57, n. 2, p. 261-264, 1992.
KUHN, C. R.; SOARES, G. J. D. Proteases e inibidores no processo de surimi. Revista Brasileira de Agrociência, v. 8, n. 1, p. 5-11, 2002.

KURADE, S. A., BARANOWSKI, J. D. Prediction of shelf-life of frozen minced fish in terms of oxidative rancidity as measured by TBARS number. Journal of Food Science, v. 52, n. 2, p. 300, 1987.

LANFER-MARQUEZ, U. M.; MIRA, N. V. M. Avaliação da composição centesimal, aminoácidos e mercúrio contaminante de surimi. Ciência e Tecnologia de Alimentos, v. 25, n. 4, p. 665-671, 2005.

LEE, C. M. Surimi manufacturing and fabrication of surimi-based products. Food Technology, p. 115-124, 1986.

MARCHI, J. F. Desenvolvimento e avaliação de produtos à base de polpa e surimi produzidos a partir de tilápia Nilótica, Oreochromis niloticus L. Viçosa, 1997, 85p. Dissertação (Mestrado em Ciência e Tecnologia de Alimentos) Universidade Federal de Viçosa (UFV).

NEIVA, C. R. P. Obtenção e caracterização de minced fish de sardinha e sua estabilidade durante a estocagem sob congelamento. São Paulo, 2003, 78p. Dissertação (Mestrado em Alimentos e Nutrição) Faculdade de Ciências Farmacêuticas da Universidade de São Paulo.

OHSHIMA, T.; SUZUKI, T.; KOIZUMI, C. New developments in surimi technology. Trends in Food Science \& Technology, v. 4, p. 157-163, 1993.

REDDY, G. V. S.; SRIKAR, L. N.; SUDHAKARA, N. S. Deteriorative change in pink perch mince during frozen storage. International Journal of Food Science and Technology, v. 27, p. 271-276, 1992.

RODRÍGUEZ, L. G. e BELLO, R. A. Elaboración de bloques congelados de pulpa de pescado y su evaluacion durante el almacenamiento. Archivos Latinoamericanos de Nutricion, v. 37, n. 2, p. 351-363, 1987.

SANTO, R. M.; COSTA, R. N.; REGULY, C. J. Preparo de alimentos prontos e quase prontos à base de pescado, para consumo institucional. Rio Grande do Sul: Fundação Universidade Rio Grande, 1980.

SAS - Statistical Analysis Systems. SAS user's guide basics Cary: SAS Institute, 1999.

SIDDAIAH, D.; REDDY, G. V. S.; RAJU, C. V.; CHANDRASEKHAR, T. C. Changes in lipids, protein and kamaboko forming ability of silver carp (Hypophthalmichthys molitrix) minced during frozen storage. Food Research International, v. 34, n. 1, p. 47-53, 2001.

SIMÕES, D. R. S.; PEDROSO, M. A.; RUIZ, W. A.; ALMEIDA, T. L. Hambúrgueres formulados com base protéica de pescado. Ciência e Tecnologia de Alimentos, v. 18, n. 4, p. 414-420, 1998.

SULTANBAWA, Y.; LI-CHAN, E. C. Y. Cryoprotective effects of sugar and polyol blends in ling cod surimi during frozen storage. Food Research International, v. 31, n. 2, p. 87-89, 1998.

SUZUKI, T. Tecnologia de las proteínas de pescado y krill. Zaragoza: Acribia, 1987.

TENUTA-FILHO, A.; JESUS, R. S. Aspectos da utilização de carne mecanicamente separada de pescado como matéria prima industrial. Boletim da Sociedade Brasileira de Ciência e Tecnologia de Alimentos, v. 37, n. 2, p. 59-64, 2003.

VYNCKE, W. Direct determination of the thiobarbituric acid value in thichloroacetic extracts of fish as a measure of oxidative rancidity. Feete-Scifen Anstrichmittel, Hamburgo, v. 72, n. 12, p. 1084-1087, 1970. 